\title{
Morphologies and prognostic significance of left ventricular volume/time curves with cardiac magnetic resonance in patients with non-ischaemic heart failure and left bundle branch block
}

\author{
Alberto Aimo ${ }^{1,2}$ - Alessandro Valleggi ${ }^{2}$ Andrea Barison ${ }^{1,2} \cdot$ Sara Salerni $^{3} \cdot$ Michele Emdin $^{1,2}$. \\ Giovanni Donato Aquaro²
}

Received: 26 December 2020 / Accepted: 13 February 2021 / Published online: 26 February 2021

(c) The Author(s) 2021

\begin{abstract}
Patients with non-ischaemic systolic heart failure (HF) and left bundle branch block (LBBB) can display a wide or narrow pattern (WP/NP) of the systolic phase of the left ventricular (LV) volume/time (V/t) curve in cardiac magnetic resonance (CMR). The clinical and prognostic significance of these patterns is unknown. Consecutive patients with non-ischaemic HF, LV ejection fraction $<50 \%$ and LBBB underwent 1.5 T CMR. Maximal dyssynchrony time (time between the earliest and latest end-systolic peaks), systolic dyssynchrony index (standard deviation of times to peak volume change), and contractility index (maximum rate of change of pressure-normalized stress) were calculated. The endpoint was a composite of cardiovascular death, HF hospitalization, and appropriate defibrillator shock. NP was found in 29 and WP in 72 patients. WP patients had higher volumes and NT-proBNP, and lower LVEF. WP patients had a longer maximal dyssynchrony time (absolute duration: $192 \pm 80$ vs. $143 \pm 65 \mathrm{~ms}, \mathrm{p}<0.001$; \% of RR interval: $25 \pm 11 \%$ vs. $8 \pm 4 \%, \mathrm{p}<0.001$ ), a higher systolic dyssynchrony index $(13 \pm 4$ vs. $7 \pm 3 \%, \mathrm{p}<0.001)$, and a lower contractility index $(2.6 \pm 1.2$ vs $3.2 \pm 1.7, \mathrm{p}<0.05)$. WP patients had a shorter survival free from the composite endpoint regardless of age, NT-proBNP or LVEF. Nonetheless, WP patients responded more often to cardiac resynchronization therapy (CRT) than those with NP (24/28 [86\%] vs. 1/11 [9\%] responders, respectively; $\mathrm{p}<0.001$ ). In patients with non-ischaemic systolic HF and LBBB, the WP of V/t curves identifies a subgroup of patients with greater LV dyssynchrony and worse outcome, but better response to CRT.
\end{abstract}

Keywords Cardiac magnetic resonance $\cdot$ Left bundle branch block $\cdot$ Dyssynchrony $\cdot$ Prognosis

\section{Background}

Cardiac magnetic resonance (CMR) provides morphologic and functional information relevant to a broad array of cardiovascular disorders. Its main qualities are excellent spatial and temporal resolution, unrestricted tomographic fields, and no exposure to ionizing radiation. CMR is the gold-standard

Alberto Aimo and Alessandro Valleggi have equally contributed to the manuscript.

Alberto Aimo

a.aimo@santannapisa.it; aimoalb@ftgm.it

1 Scuola Superiore Sant'Anna, Piazza Martiri della Libertà 33, 56124 Pisa, Italy

2 Fondazione Toscana Gabriele Monasterio, Piazza Martiri della Libertà 33, 56124 Pisa, Italy

3 University Hospital of Chieti, Chieti, Italy technique for the quantification of left ventricular (LV) volumes, and offers a variety of alternative applications for the assessment of both systolic and diastolic function, some of them superior to echocardiography in accuracy and reproducibility, other complementary $[1,2]$.

Several techniques for the evaluation of LV dyssynchrony by CMR have been proposed [3]. Conventional analysis of the short-axis, balanced steady-state free precession (bSSFP) acquisition allows to calculate LV volume/time curves and their first derivative $\mathrm{dV} / \mathrm{dt}$ across all cardiac phases; cardiac dyssynchrony can be assessed by visualizing how steep the ventricular emptying is and how it is distributed throughout systole. Sohal et al. introduced the systolic dyssynchrony index, defined as the standard deviation of the regional times to peak volume change from segmental volume/time (V/t) curves [4]. Further analyses may be performed by tracking myocardial deformation during the cardiac cycle and calculating systolic strain and strain rate. Feature tracking analysis 
of bSSFP imaging detects anatomical features of interest in the LV subendocardium and subepicardium along the cardiac cycle, similarly to echocardiographic speckle tracking $[5,6]$. A second technique is cardiac tagging, which is based on the application of a specific radiofrequency pulse at baseline to mark several lines or grids in the myocardium, which can then be followed over time; it and has been validated against sonomicrometry measurements [7]. A circumferential uniformity ratio estimate (CURE) derived from myocardial tagging has been proposed [8]. A third technique relies on phase contrast imaging, which can be used to track myocardial movements in any direction with almost the same frame rates as echocardiography. This technique remains to be properly validated, as a close relationship with echocardiographic and hemodynamic data was demonstrated only in small studies $[9,10]$. Other techniques to calculate strain and strain rate have been investigated [11, 12].

Among these possible approaches to the assessment of LV dyssynchrony, V/t curves are particularly promising because they can be automatically generated from standard bSSFP images by common post-processing CMR software. $\mathrm{V} / \mathrm{t}$ curves visually represent the changes in LV volumes throughout the cardiac cycle, and could be potentially used to perform a quantitative assessment of both systolic and diastolic function [13]. We observed that, in patients with non-ischemic HF and left bundle branch block (LBBB), the systolic phase of the V/t curve can display two patterns: a narrow pattern (NP), similar to the profile of subjects with no LBBB, and a wide pattern (WP), showing an irregular profile and multiple peaks (Fig. 1). This study aimed to define the clinical correlates of the WP pattern, its impact on patient outcome, and on the response to CRT.

\section{Methods}

\section{Study population}

Consecutive patients with non-ischaemic systolic HF (LVEF $<50 \%)$ and LBBB evaluated in the outpatient clinic of a tertiary referral centre for HF (Fondazione Toscana Gabriele Monasterio, Pisa, Italy) were referred to CMR examination at the same Institution. CMR was requested as part of a comprehensive screening of chronic HF patients (see below). The inclusion criteria were: a diagnosis of HF according to European Society of Cardiology Guidelines $[14,15]$; clinical stability and no changes in therapy since $\geq 3$ months; non-ischaemic aetiology; LBBB; no
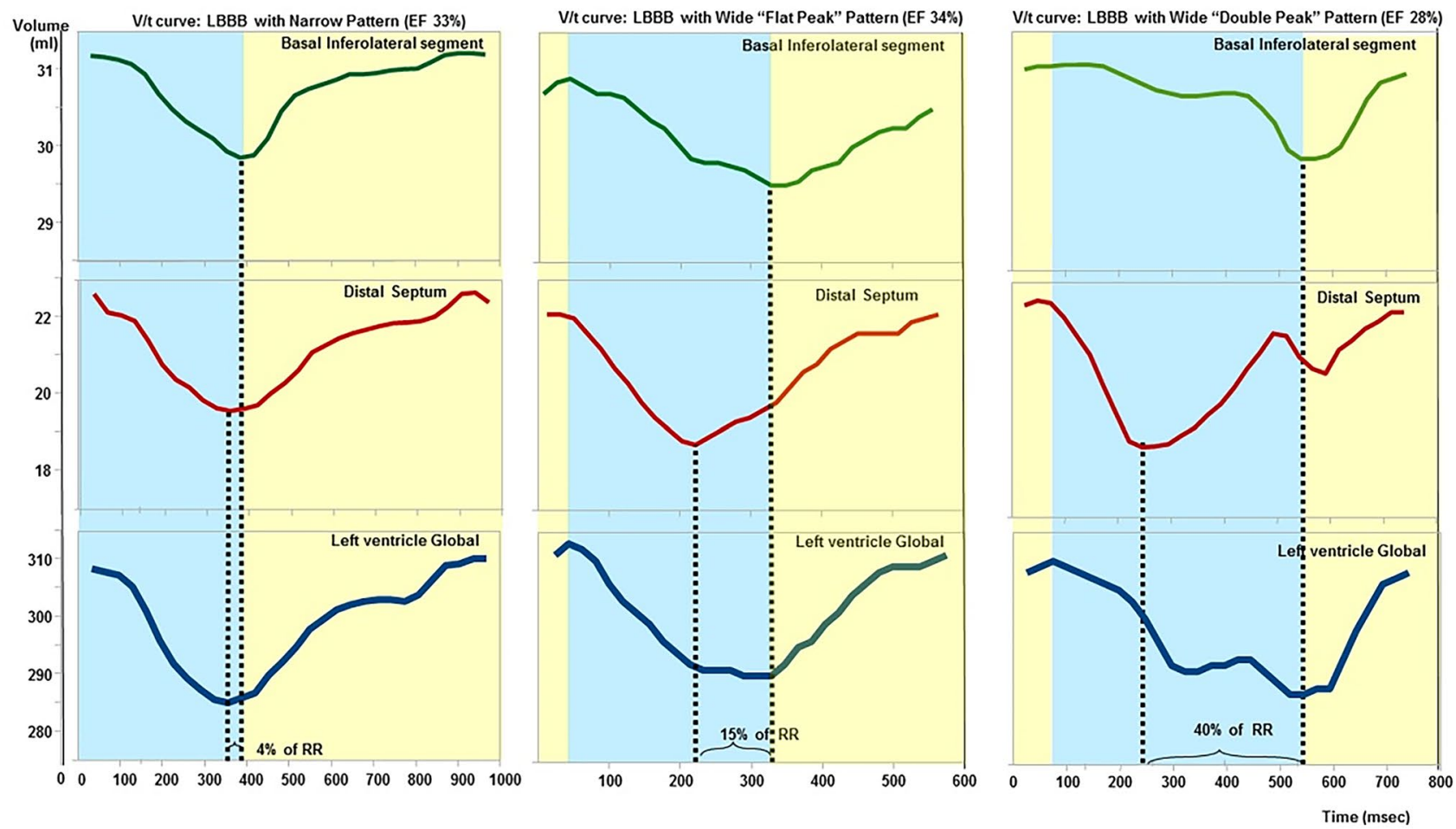

Fig. 1 Examples of narrow pattern and wide pattern. The volume/ time (V/t) curves of 3 patients with non-ischaemic systolic heart failure are reported. The regional $\mathrm{V} / \mathrm{t}$ curve of basal inferolateral region and distal septum are shown in the upper and middle panel, respectively. The panel below provides the global left ventricular V/t curves. The maximal dyssynchrony time was $4 \%$ of the R-R interval in the patient with the narrow pattern (left), $15 \%$ in the patient with the wide, "flat-peak" pattern (centre), and $40 \%$ in the patient with the wide, "double-peak" pattern (right) 
contraindications to CMR examination. All patients underwent coronary angiography to establish the non-ischaemic aetiology, in agreement with the World Health Organization definition ("a dilated cardiomyopathy with impaired contractile performance not explained by the extent of coronary artery disease or ischemic damage") [16]. Exclusion criteria were: age $<18$ years, congenital heart disease, recent myocarditis ( $<6$ months), peripartum cardiomyopathy, arrhythmogenic right ventricular cardiomyopathy, severe primary valve disease, untreated hypertension, hypertrophic cardiomyopathy, cardiac amyloidosis, estimated glomerular filtration rate $<30 \mathrm{~mL} / \mathrm{min} / 1.73 \mathrm{~m}^{2}$, and contraindications to CMR (severe claustrophobia, presence of CMR-unsafe metal devices). Furthermore, patients with "benign" aetiologies such as enolic, Tako-tsubo or tachymocardiopathies were excluded. LBBB was diagnosed through standard, 12-lead electrocardiogram (ECG), following established criteria: QRS duration $\geq 130 \mathrm{~ms}$; QS or $\mathrm{rS}$ in lead V1; broad (frequently notched or slurred) R waves in leads I, aVL, V5, or V6; and absent q waves in leads V5 and V6 [17].

101 patients were prospectively enrolled from 2013 to 2015. In addition to 12-lead ECG and CMR (see below), these patients underwent:

- Transthoracic echocardiogram, performed and interpreted according to current Guidelines [18-22];

- Laboratory evaluation: full blood cell count, C-reactive protein, creatinine, transaminases, thyroid hormones, cardiac troponin I, N-terminal pro-B-type natriuretic peptide (NT-proBNP), resting norepinephrine (NE), plasma renin activity, and aldosterone levels) [23];

- Symptom-limited cardiopulmonary exercise testing (CPET) on a bicycle ergometer (Vmax, Sensormedics, Yorba Linda, CA, USA) [24].

- 24-hour ECG Holter recording by a three-lead (precordial, posterior, inferior leads) digital system (Elamedical, France). At Holter monitoring, ventricular ectopic beats (VEBs) were considered relevant if they were grade IV or V on the Lown scale [25].

Informed consent was obtained from each patient. The study protocol conformed to the ethical guidelines of the 1975 Declaration of Helsinki [26], and was approved by the Institution's human research committee. Patients were compared with 20 age- and sex-matched healthy controls, undergoing CMR with no contrast medium administration.

\section{Cardiac magnetic resonance}

Patients underwent CMR with an 8-channel phased-array surface receiver coil and vectorcardiogram triggering using a 1.5 T scanner (Signa Excite, GE Healthcare, Milwaukee, USA). Biventricular systolic function was assessed by breath-hold steady-state free precession cine imaging in the short-axis (SA) stack (8-mm thickness, no gap). Sequence parameters were: field-of-view: $360-400 \mathrm{~mm}$, repetition/ echo time: $3.2 / 1.6 \mathrm{~ms}$, flip angle: $45-60^{\circ}$, matrix: $224 \times 224$, phases: 30 . Late gadolinium enhancement (LGE) imaging was performed between 10 and 20 min after contrast agent administration (Gadoteric acid, DOTAREM, $0.2 \mathrm{mmol} /$ $\mathrm{kg}$ ) using a segmented T1-weighted gradient-echo inversion-recovery pulse sequence. In SA orientation, the LV was encompassed by contiguous 8 -mm thick slices (with no inter-slice gap). Inversion time (TI) was individually adapted to suppress the signal of normal remote myocardium $(220-320 \mathrm{~ms})$. Sequence parameters were: field-ofview: $360-400 \mathrm{~mm}$, slice thickness: $8 \mathrm{~mm}$, repetition/echo time: $4.6 / 1.3 \mathrm{~ms}$, flip angle: $15-20^{\circ}$, matrix: $224 \times 192$.

All CMR studies were analysed off-line on the Advantage Workstation (GE Healthcare, Milwaukee, USA) with a dedicated software (MASS 6.1, Medis, Leiden, Netherlands) by an experienced CMR reader (A.B.) blinded to all other patient data. $\mathrm{LV}$ and RV volumes, mass and global function were calculated on SA cine images.

LV volumes were measured in each cardiac phase and plotted as a function of time to generate a V/t curve (Fig. 1). The pattern of the systolic phase of global V/t curves were determined by 2 independent expert CMR readers (G.D.A. and A.B.) blinded to all other CMR and clinical data. The NP consisted in a progressive, rapid reduction of LV volume with a well-defined, smooth peak, closely recapitulating the changes of volumes during systole. Conversely, the WP could display the following morphologies: "double peak" (two systolic peaks), "saw-tooth" (multiple peaks) or "flat" (large systolic plateau) (Fig. 1). A perfect inter-observer agreement $(\mathrm{k}=1)$ was found in the identification of the WP or NP pattern. Even the intra-observer agreement during 2 repeated examinations 1-month apart was complete $(\mathrm{k}=1)$. All healthy controls were evaluated in the same blinded fashion and found to have a NP.

The presence and extent of LGE were determined on short-axis images by detecting areas of myocardium with signal intensity $\geq 6$ standard deviations above remote, nonenhanced myocardium $[27,28]$. The maximal $\mathrm{dV} / \mathrm{dt}$ ratio during the systolic phase and the contractility index (maximum rate of change of pressure-normalized stress: $d \sigma^{*} /$ $\mathrm{dt}_{\max }$, where $\sigma^{*}=\sigma / \mathrm{P}$, and $\sigma$ and $\mathrm{P}$ are circumferential stress and pressure, respectively) were calculated as indices of $\mathrm{LV}$ contractility [29].

For a regional analysis of LV systolic kinesis, the endocardial contours of all cardiac phases was divided into 6 equiangular segments at the basal and mid-ventricular levels and 4 equiangular segments at the distal level; the systolic dyssynchrony index was calculated as the standard deviation of the regional times to peak volume change [4]. V/t curves were derived in $16 \mathrm{LV}$ regions (according to the American 
Heart Association/American College of Cardiology segmentation and excluding LV apex) [30], and the end-systolic peak was identified in each regional V/t curve. Maximal dyssynchrony time was defined as the temporal difference between the end-systolic peaks of the segments with the earliest and the latest peak; it was expressed both in seconds and as a percentage of the RR interval.

\section{Follow-up}

All patients received optimal medical therapy, and CRT with defibrillation (CRT-D) when indicated [14, 15]. Results of CMR examination were not used to guide lead placement. In the absence of standardized criteria [31], the response to CRT was defined as improvement in at least 1 New York Heart Association (NYHA) class together with any increase in LVEF and $\geq 10 \%$ decrease in LV end-systolic volume at TTE after 3 months. The outcome status was assessed in December 2020 based on electronic health records (EHRs) or phone interviews with patients, relatives, or general practitioners; specifically, phone interviews were performed for all patients who did not have adverse outcomes recorded in the EHRs. The endpoint was a composite of cardiovascular death, HF hospitalization, or appropriate defibrillator shock; patients were censored at the time of the first event.

\section{Statistical analysis}

Statistical analysis was performed using IBM SPSS Statistics (version 22, 2013). Normal distribution was assessed through the Kolmogorov-Smirnov test; variables with normal distribution were presented as mean \pm standard deviation, while those with non-normal distribution as median and interquartile interval. Differences between groups were tested through the Mann-Whitney U test, and categorical variables were compared by the Chi-square test with Yates correction. In Kaplan-Meier analysis, survival was compared through the log-rank test (Mantel-Cox). Predictors of the composite endpoints were searched through univariate and bivariate Cox regression analysis; the "one-in-ten" rule was followed to avoid model overfitting [32]. Two-tailed $p$ values $<0.05$ were considered as significant.

\section{Results}

\section{Population characteristics and correlates of the two V/t curve morphologies}

Patients $(\mathrm{n}=101)$ were aged $66 \pm 11$ years, $55 \%$ were males, and LVEF at CMR was 29\% (25-35). The estimated glomerular filtration rate (eGFR) was $60 \mathrm{~mL} / \mathrm{min} / 1.73 \mathrm{~m}^{2}$ (46-80), and NT-proBNP levels were 1,060 ng/L (445-1994).
Patients were on optimal medical therapy with beta-blockers, angiotensin-converting enzyme inhibitors or angiotensin-receptor blockers, mineralocorticoid receptor antagonists (MRA), in the absence of contraindications (Table 1). No patient had a device at baseline, and all patients were in sinus rhythm.

The systolic phase of the V/t curve displayed a NP in 29 patients, and a WP in 72. Mean QRS duration did not differ significantly between patients with a NP and those with a WP. However, patients with a WP had a higher heart rate, more frequent high-grade VEBs, and a more prominent neurohormonal activation, with markedly higher plasma NTproBNP and norepinephrine (Table 1). End-systolic and enddiastolic diameters at transthoracic echocardiogram were greater in the WP group, while the grades of mitral regurgitation did not display significant differences (Table 2).

\section{CMR findings}

The numbers of patients with WP or NP are reported above; all healthy controls (mean age 64 years, 50\% men) displayed a V/t curve morphology close to the NP. Patients with a WP had significantly greater LV indexed volumes and a lower LVEF, but no significant differences in terms of LGE prevalence, extent, or pattern (Table 2).

The WP pattern was associated with a longer maximal dyssynchrony time (absolute duration: $192 \pm 80$ vs. $143 \pm 65 \mathrm{~ms}, \mathrm{p}<0.001 ; \%$ of RR interval: $25 \pm 11 \%$ vs. $8 \pm 4 \%, \mathrm{p}<0.001)$. Even the systolic dyssynchrony index was higher in WP patients $(13 \pm 4$ vs. $7 \pm 3 \%, \mathrm{p}<0.001)$. The contractility index was lower in WP patients $(2.6 \pm 1.2$ vs $3.2 \pm 1.7, \mathrm{p}=0.045)$.

\section{Patterns of $\mathrm{V} / \mathrm{t}$ curve for outcome prediction}

Over a median 3.7-year follow-up (2.0-4.9), 7 patients died, 6 of them for cardiovascular causes. Eleven patients had defibrillator shocks because of life-threatening ventricular arrhythmias, and 15 patients were hospitalized because of worsening HF. Overall, the composite endpoint of cardiovascular death, HF hospitalization or appropriate defibrillator discharge occurred in 29 patients.

Patients with a WP had a significantly shorter survival free from the composite endpoint (Fig. 2). In other words, patients with a WP were more likely to experience an event during follow-up than those with a NP (Fig. 3). Among all characteristics listed in Tables 1 and 2, the following univariate predictors emerged: age, eGFR, NT-proBNP, LVESD at echo, LVEF at CMR, LGE presence, and WP (Table 3). The WP displayed an independent prognostic value from all the other univariate predictors, analysed by separate bivariate analysis because of the low number of events $(n=29$; Table 4$)$. Among the other variables, WP retains independent prognostic significance 
Table 1 Population characteristics

\begin{tabular}{lllll}
\hline & All patients $\mathrm{n}=101$ & Narrow pattern $\mathrm{n}=29$ & Wide pattern $\mathrm{n}=72$ & $\mathrm{p}$ \\
\hline Age (years) & $66 \pm 11$ & $63 \pm 14$ & $67 \pm 9$ & 0.314 \\
Men, $\mathrm{n}(\%)$ & $55(55)$ & $15(52)$ & $40(56)$ & 0.726 \\
BMI $\left(\mathrm{kg} / \mathrm{m}^{2}\right)$ & $26 \pm 4$ & $26 \pm 4$ & $26 \pm 4$ & 0.991 \\
LVEF CMR (\%) & $29(25-35)$ & $32(28-39)$ & $28(25-33)$ & $\mathbf{0 . 0 1 5}$ \\
NYHA I/II-III-IV, n (\%) & $7 / 94(7 / 93)$ & $1 / 28(3 / 97)$ & $6 / 66(8 / 92)$ & 0.380 \\
VEB grade IV-V Lown, n (\%) & $52(51)$ & $9(33)$ & $43(59)$ & $\mathbf{0 . 0 0 3}$ \\
Diabetes, n (\%) & $24(24)$ & $1(3)$ & $23(32)$ & $\mathbf{0 . 0 0 2}$ \\
Hypertension, n (\%) & $58(57)$ & $15(52)$ & $43(60)$ & 0.462 \\
Hypercholesterolemia, $\mathrm{n}(\%)$ & $35(35)$ & $9(31)$ & $26(36)$ & 0.628 \\
QRS width (ms) & $150(140-161)$ & $150(140-160)$ & $150(140-165)$ & 0.468 \\
Heart rate (b.p.m.) & $68(60-78)$ & $65(57-74)$ & $70(62-79)$ & $\mathbf{0 . 0 4 5}$ \\
Haemoglobin (g/dL) & $13(13-14)$ & $13(13-15)$ & $13(12-14)$ & 0.226 \\
eGFR (mL/min/1.73 m $\left.{ }^{2}\right)$ & $60(46-80)$ & $72(51-82)$ & $60(43-80)$ & 0.252 \\
NT-proBNP (ng/L) & $1,060(450-1,994)$ & $493(317-1,740)$ & $1,338(557-2,624)$ & $\mathbf{0 . 0 0 3}$ \\
NE (ng/L) & $362(270-585)$ & $289(202-412)$ & $441(304-625)$ & $\mathbf{0 . 0 0 3}$ \\
PRA (ng/mL/h) & $1.4(0.4-3.7)$ & $1.2(0.4-2.9)$ & $1.4(0.3-4.1)$ & 0.731 \\
Aldosterone (ng/L) & $142(76-224)$ & $169(80-220)$ & $131(74-225)$ & 0.371 \\
VO $/$ /kg (mL/kg/min) & $15(12-17)$ & $15(12-18)$ & $14(12-17)$ & 0.722 \\
VE/VCO 2 & $30(28-36)$ & $30(28-35)$ & $31(28-36)$ & 0.541 \\
Beta-blocker, n (\%) & $988(97)$ & $27(93)$ & $71(99)$ & 0.140 \\
ACEi/ARB, n (\%) & $95(94)$ & $29(100)$ & $66(92)$ & 0.109 \\
MRA, n (\%) & $80(79)$ & $22(76)$ & $58(81)$ & 0.824 \\
\hline
\end{tabular}

Significant $\mathrm{p}$ values are reported in bold

Variables with normal distribution were presented as mean \pm standard deviation, while those with non-normal distribution as median and interquartile interval

$A C E i$ angiotensin-converting enzyme inhibitor; $A R B$ angiotensin receptor blocker; $B M I$ body mass index; $L B B B$ left bundle branch block; $M R A$ mineralocorticoid receptor antagonist; $N E$ norepinephrine; $N T$ proBNP N-terminal pro-B-type natriuretic peptide; NYHA New York Heart Association; PRA plasma renin activity; $P V C$ premature ventricular complex; $V E / V C \mathrm{O}_{2}$ ventilation/carbon dioxide output; $V E B$ ventricular ectopic beat; $\mathrm{VO}_{2}$ oxygen consumption when evaluated against diabetes (HR 4.82, 95\% CI 1.12-20.68, $\mathrm{p}=0.034$ ), which was significantly prevalent in the WP cohort (Table 1), and predicts worse outcomes in patients with HF [33].

\section{Patterns of $\mathrm{V} / \mathrm{t}$ curve and the prediction of response to CRT}

After baseline CMR, 39 patients (28 with a WP, 11 with a NP) underwent CRT implantation (which in all cases was a CRT-D device). Despite their worse prognosis, patients with a WP responded more often to CRT than those with a NP (24/28 [86\%] vs. $1 / 11[9 \%]$ responders, respectively; $p<0.001)$. The prognostic benefit from CRT was evident also in the WP group, where patients on CRT had a better outcome ( $\mathrm{p}=0.020$ at Kaplan-Meier analysis).

\section{Discussion}

In patients with non-ischaemic systolic HF (LVEF < 50\%) and LBBB, the systolic phase of the V/t curve showed 2 markedly different morphologies, namely a WP or NP. Patients with a WP displayed a worse HF status, more frequent high-grade VEBs, and a greater neurohormonal activation than those with a NP. The WP was also associated with more severe dyssynchrony and lower LV contractility. Patients with a WP had also a shorter survival free from cardiovascular events. Finally, among patients undergoing 
Table 2 Imaging findings in patients with narrow and wide pattern

\begin{tabular}{|c|c|c|c|c|}
\hline & All patients $n=101$ & Narrow pattern $n=29$ & Wide pattern $n=72$ & $\mathrm{p}$ \\
\hline \multicolumn{5}{|l|}{ TTE } \\
\hline LVEF (\%) & $30(25-35)$ & $32(28-39)$ & $28(25-33)$ & $<0.001$ \\
\hline LVEDD (mm) & $65(59-70)$ & $59(54-66)$ & $66(60-70)$ & 0.001 \\
\hline $\operatorname{LVESD}(\mathrm{mm})$ & $54(49-60)$ & $50(44-55)$ & $55(49-61)$ & $<0.001$ \\
\hline $\mathrm{E} / \mathrm{e}$ & $12(9-16)$ & $11(9-16)$ & $12(9-17)$ & 0.535 \\
\hline $\begin{array}{l}\text { MR grades (mild, moder- } \\
\text { ate, moderate-severe, } \\
\text { severe) }\end{array}$ & $48,38,7,8$ & $15,10,4,0$ & $33,28,3,8$ & 0.100 \\
\hline $\mathrm{n}(\%)$ & $(48,38,7,8)$ & $(52,45,13,0)$ & $(46,39,4,11)$ & \\
\hline TAPSE $(\mathrm{mm})$ & $20(17-23)$ & $19(17-23)$ & $20(18-23)$ & 0.737 \\
\hline \multicolumn{5}{|l|}{ CMR } \\
\hline $\operatorname{LVEF}(\%)$ & $26(21-34)$ & $33(27-40)$ & $23(19-29)$ & $<0.001$ \\
\hline LVEDVi $\left(\mathrm{mL} / \mathrm{m}^{2}\right)$ & $118(92-145)$ & $97(58-131)$ & $119(98-150)$ & $\mathbf{0 . 0 1 3}$ \\
\hline $\operatorname{LVESVi}\left(\mathrm{mL} / \mathrm{m}^{2}\right)$ & $86(662-111)$ & $72(45-90)$ & $93(82-116)$ & 0.002 \\
\hline LGE & $52(51)$ & $12(41)$ & $40(56)$ & 0.192 \\
\hline LGE extent ( $\%$ of LV mass) & $2(1.8-4.2)$ & $2.0(1.8-3.1)$ & $2.1(1.4-4.2)$ & 0.080 \\
\hline Subepicardial LGE, n (\%) & $6(12)$ & $2(17)$ & $4(10)$ & 0.790 \\
\hline Mid-wall LGE, n (\%) & $46(88)$ & $10(83)$ & $36(90)$ & 0.500 \\
\hline Septal LGE, n (\%) & $15(29)$ & $3(25)$ & $12(30)$ & 0.740 \\
\hline
\end{tabular}

Significant $\mathrm{p}$ values are reported in bold

As all variables had a non-normal distribution, they were presented as median and interquartile interval

$C M R$ cardiac magnetic resonance; $L G E$ late gadolinium enhancement; $L V E D D$ left ventricular end-diastolic diameter; $L V E D V i$ left ventricular end-diastolic volume indexed; $L V E F$ left ventricular ejection fraction; $L V E S D$ left ventricular end-systolic diameter; $L V E S V i$ left ventricular end-systolic volume indexed; TAPSE tricuspid annular plane systolic excursion; TTE transthoracic echocardiogram

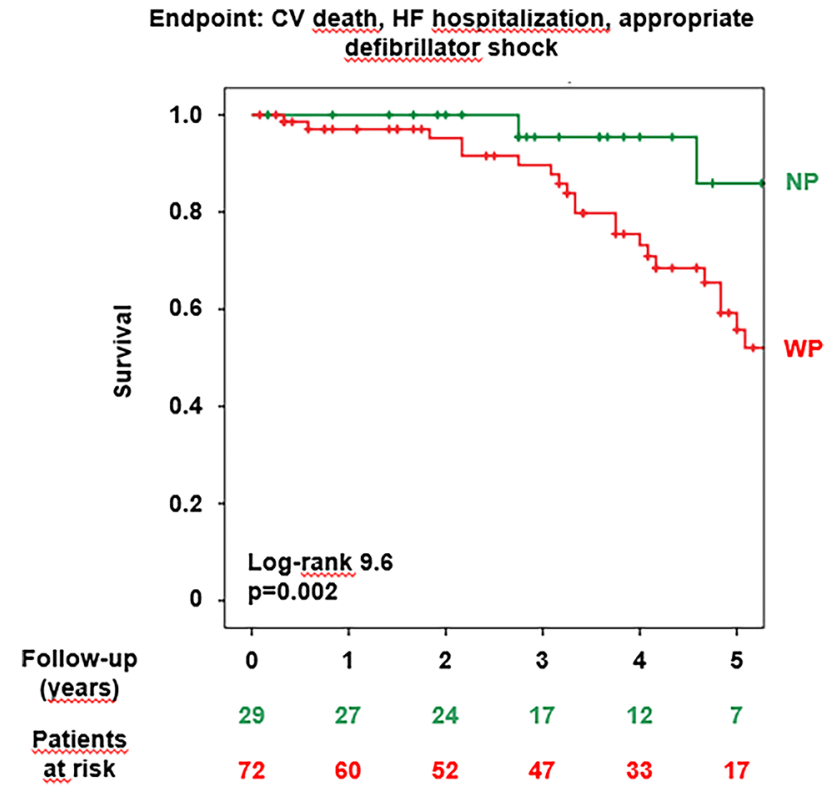

Fig. 2 Patterns of the volume/time curve and event-free survival. $C V$ cardiovascular; $H F$ heart failure; $N P$ narrow pattern; $W P$ wide pattern

CRT implantation during follow-up, patients with a WP were more likely to respond to CRT.
An LBBB is found in about $20 \%$ of patients with HF [34], and is an expression of structural impairment of the myocardium. LBBB contributes to LV dysfunction by negatively affecting perfusion, systolic function and diastolic relaxation [35, 36]. Interestingly, studies exploring the clinical and prognostic correlates of LBBB have almost constantly performed a cumulative assessment of patients with this conduction disorder, despite the extreme variability in QRS duration and morphology, which suggests a similar heterogeneity in the determinants and consequences of LBBB [37, 38].

In this study we selected patients with non-ischemic cardiomyopathy to avoid the confounding factor of regional scarring altering mechanical contraction. We describe for the first time that the systolic phase of the V/t curve can display 2 morphologies, i.e. a NP or a WP. All patients had a LBBB, then a significantly impaired propagation of action potentials. Different patterns of impulse propagation may result in different morphologies of the V/t curve, without necessarily affecting total QRS duration. The NP demonstrates a relative preservation of the normal sequence of $\mathrm{LV}$ activation, while the WP is characterized by a markedly dyssynchronous LV contraction.

In our cohort, $29 \%$ of patients displayed a NP, and $71 \%$ a WP. The systolic dyssynchrony index was significantly 
Patient 1: $M, 65$ years

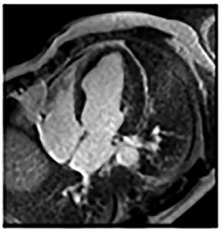

LGE
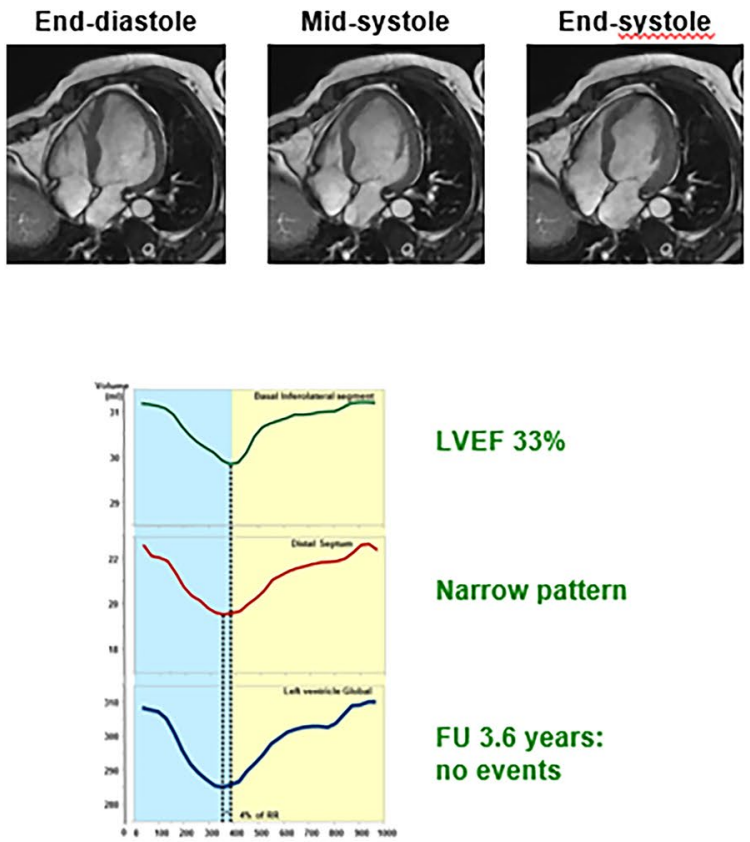

LVEF $33 \%$

Narrow pattern

FU 3.6 years:

no events

Fig. 3 Patterns of the volume/time curve and risk of events. A 65-year-old man (patient 1, left) and a 67-year-old woman (patient 2, right) are presented. Late gadolinium enhancement (LGE) images showed small areas of mid-wall fibrosis in the interventricular septum in both cases, together with some subepicardial LGE in the lateral wall in patient 2 . For each patient, 4-chamber acquisitions cor-

higher in patients with WP. Accordingly, maximal dyssynchrony time was higher in patients with WP, on average $25 \%$ of the cardiac cycle, compared with $8 \%$ in patients with NP. In other words, ventricular activation was completed in a quarter of the entire cardiac cycle, compared with $8 \%$ of the cardiac cycle in patients with NP. Not surprisingly, LV systolic function was less effective in the WP subgroup, as demonstrated by a lower contractility index. In parallel, WP patients displayed greater diameters and volumes of the LV, demonstrating a more pronounced LV remodelling. An apparent discrepancy remains between the worse clinical outcome and the more frequent CRT response in WP patients compared to NP patients: it is possible that CRT might counteract disease progression in WP patients, but not enough to make their prognosis similar to NP patients. Further studies might address the differential prognostic impact of CRT in NP and WP patients.
Patient $2: W, 67$ years
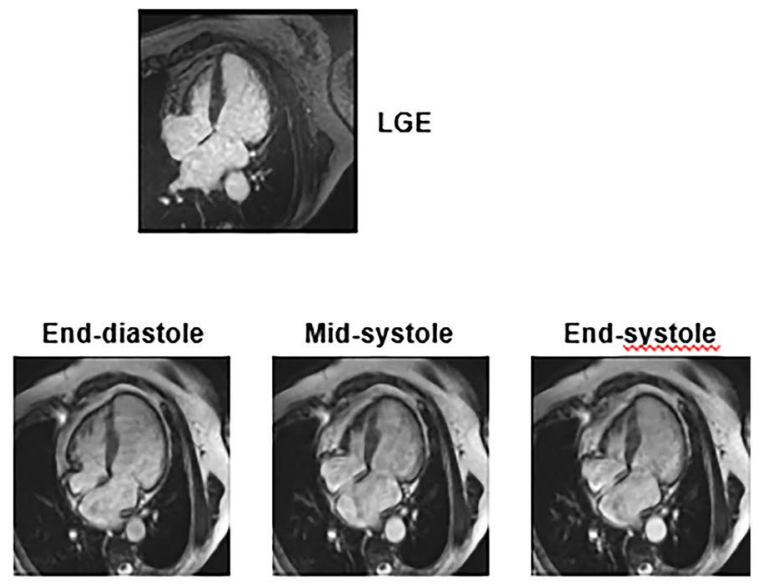

LVEF $34 \%$

Wide

pattern

FU 3.8 years: combined endpoint

responding to end-diastole, mid-systole and end-systole are reported. Left ventricular ejection fraction (LVEF) and follow-up (FU) duration were similar, and LV end-diastolic volumes did not differ significantly (data not shown). Still, patient 1 had a narrow pattern and did not experience any event, while patient 2 had a wide pattern and had an event (heart failure hospitalization)

CMR-assessed myocardial fibrosis has been repeatedly considered as a useful tool for risk stratification, and as a guide to treatment [39-41]. Different patterns of myocardial fibrosis could explain heterogeneity in LV conduction, manifesting as WP or NP. Nevertheless, no significant differences were found between WP and NP with regard to LGE presence; among patients with LGE, its extent or distribution pattern did not differ between WP and NP patients. Different LBBB morphologies thus seem to be related to a higher degree of LV remodelling rather than to different patterns of fibrosis, even though further larger studies are needed to address this point.

Calculation of another index of dyssynchrony, namely the CURE [8], requires complex post-processing which is not always available in CMR laboratories. Visual assessment of the systolic phase of the V/t curve may be a valuable and more viable alternative to these indices. Even the 
Table 3 Univariate predictors of the composite outcome

\begin{tabular}{|c|c|c|c|}
\hline & $\mathrm{p}$ & HR & $95 \% \mathrm{CI}$ \\
\hline Age & 0.008 & 1.06 & $1.02-1.11$ \\
\hline Men & 0.444 & - & - \\
\hline BMI & 0.840 & - & - \\
\hline NYHA I/II-III & 0.280 & - & - \\
\hline VEB grade IV-V Lown & 0.991 & - & - \\
\hline Diabetes & 0.602 & - & - \\
\hline Hypertension & 0.294 & - & - \\
\hline Hypercholesterolemia & 0.306 & - & - \\
\hline QRS width & 0.263 & - & - \\
\hline Heart rate & 0.064 & - & - \\
\hline Haemoglobin & 0.278 & - & - \\
\hline eGFR & 0.021 & 0.98 & $0.97-0.99$ \\
\hline NT-proBNP & 0.047 & 1.01 & $1.00-1.01$ \\
\hline $\mathrm{NE}$ & 0.547 & - & - \\
\hline PRA & 0.614 & - & - \\
\hline Aldosterone & 0.059 & - & - \\
\hline $\mathrm{VO}_{2} / \mathrm{kg}$ & 0.406 & - & - \\
\hline $\mathrm{VE} / \mathrm{VCO}_{2}$ & 0.779 & - & - \\
\hline Beta-blocker & 0.252 & - & - \\
\hline ACEi/ARB & 0.054 & - & - \\
\hline MRA & 0.919 & - & - \\
\hline \multicolumn{4}{|l|}{ TTE } \\
\hline LVEF & 0.052 & - & - \\
\hline LVEDD & 0.338 & - & - \\
\hline LVESD & 0.045 & 1.01 & $1.01-1.02$ \\
\hline E/e' & 0.591 & - & - \\
\hline TAPSE & 0.414 & - & - \\
\hline \multicolumn{4}{|l|}{ CMR } \\
\hline LVEF & 0.048 & 0.97 & $0.92-0.99$ \\
\hline LVEDVi & 0.712 & - & - \\
\hline LVESVi & 0.608 & - & - \\
\hline LGE & 0.049 & 2.16 & $1.01-4.66$ \\
\hline LGE extent & 0.055 & - & - \\
\hline $\begin{array}{l}\text { Subepicardial/mid-wall/ } \\
\text { septal LGE }\end{array}$ & 0.219 & - & - \\
\hline WP & $\mathbf{0 . 0 3 3}$ & 4.81 & $1.14-20.37$ \\
\hline
\end{tabular}

Significant $\mathrm{p}$ values are reported in bold

Results from univariate Cox regression analysis are reported

$A C E i$ angiotensin-converting enzyme inhibitor; $A R B$ angiotensin receptor blocker; $B M I$ body mass index; $C M R$ cardiac magnetic resonance; $L B B B$ left bundle branch block; $L G E$ late gadolinium enhancement; $L V E D D$ left ventricular end-diastolic diameter; $L V E D V i$ left ventricular end-diastolic volume indexed; $L V E F$ left ventricular ejection fraction; $L V E S D$ left ventricular end-systolic diameter; $L V E S V i$ left ventricular end-systolic volume indexed; $M R A$ mineralocorticoid receptor antagonist; $N E$ norepinephrine; $N T$-proBNP N-terminal proB-type natriuretic peptide; NYHA New York Heart Association; PRA plasma renin activity; $P V C$ premature ventricular complex; TAPSE tricuspid annular plane systolic excursion; TTE transthoracic echocardiogram; $V E / V C O_{2}$ ventilation/carbon dioxide output; $V E B$ ventricular ectopic beat; $V \mathrm{O}_{2}$ oxygen consumption; $W P$ wide pattern post-processing of tagging acquisitions is very complex and time consuming, whereas the $\mathrm{V} / \mathrm{t}$ curves are automatically generated in the majority of current post-processing software for CMR without the need for regional evaluation. We found that WP was associated to higher systolic dyssynchrony index than the NP, confirming that the shape of $\mathrm{V} / \mathrm{t}$ curve may be a simple way to detect mechanical dyssynchrony. Our method is simple, highly reproducible and commonly available because it requires only bSSFP short-axis images.

Several limitations must be acknowledged. First, this was a small, single-centre study, and confirmation on larger patient cohorts is warranted. Furthermore, although patients were managed according to Guideline recommendations, a limited number of patients received a CRT device [14]. This did not allow to perform a comprehensive assessment of predictors of response to CRT, including for example the presence and extent of myocardial fibrosis, or the correlates of such response, such as the changes in mitral regurgitation severity. Second, subgroup analyses considering specific aetiologies (including for example genetic dilated cardiomyopathy) were not performed. Third, temporal resolution is crucial to assess mechanical dyssynchrony, and CMR has a lower temporal resolution (ranging between 40 and $50 \mathrm{~ms}$ ) than transthoracic echocardiography ( $<10 \mathrm{~ms})$. CMR assessment of dyssynchrony was not compared with an asynchrony study performed with an echocardiogram, which seems to predict response to CRT and outcome [42]. In addition to this comparison, further studies should examine the added value of a CMR assessment of dyssynchrony over an echocardiographic evaluation. Fourth, the small number of events required that a composite outcome measure be chosen, instead of single outcomes. Fifth, information from T1-mapping analysis was not available, although quantitative LGE evaluation allowed to search for the most evident and prognostically meaningful manifestation of an expansion of myocardial extracellular volume, i.e., myocardial scarring. Sixth, the possibility to draw conclusion with regard to the prediction of response to CRT is limited by the low number of patients.

In conclusion, in patients with non-ischaemic $\mathrm{HF}$ and LBBB, the presence of a WP in the systolic phase of the $\mathrm{V} / \mathrm{t}$ curve identifies a subgroup of patients with greater LV dyssynchrony and a worse cardiac outcome, but a more frequent response to CRT. The systolic pattern of $\mathrm{V} / \mathrm{t}$ curve might be used as a novel marker of mechanical dyssynchrony, and as a predictor of response to CRT. 
Table 4 Independent prognostic value of the wide pattern (WP) for the prediction of cardiovascular death, heart failure hospitalization, appropriate defibrillator shock: bivariate Cox regression analysis

\begin{tabular}{|c|c|c|c|c|c|c|}
\hline & Age & eGFR & NT-proBNP & LVESD & LVEF & LGE \\
\hline \multirow[t]{6}{*}{ WP } & $\begin{array}{l}\mathrm{p}=0.015 \\
\mathrm{HR} 1.07 \\
(95 \% \mathrm{CI} \\
1.01-1.12)\end{array}$ & - & - & - & - & - \\
\hline & - & $\begin{array}{l}\mathrm{p}=0.025 \\
\mathrm{HR} 5.37 \\
(95 \% \mathrm{CI} \\
1.24-23.30)\end{array}$ & - & - & - & - \\
\hline & - & - & $\begin{array}{l}p=0.012 \\
\text { HR } 6.44 \\
(95 \% \text { CI } \\
1.52-27.37)\end{array}$ & - & - & - \\
\hline & - & - & - & $\begin{array}{l}\mathrm{p}=0.024 \\
\mathrm{HR} 6.02 \\
(95 \% \mathrm{CI} \\
1.27-28.65)\end{array}$ & - & - \\
\hline & - & - & - & - & $\begin{array}{l}\mathrm{p}=0.044 \\
\text { HR 5.42 } \\
(95 \% \text { CI } \\
1.04-28.18)\end{array}$ & - \\
\hline & - & - & - & - & - & $\begin{array}{l}\mathrm{p}=0.011 \\
\mathrm{HR} 13.47 \\
(95 \% \mathrm{CI} \\
1.81-100.08)\end{array}$ \\
\hline
\end{tabular}

$\mathrm{p}$ values, hazard ratio (HR) and the corresponding 95\% confidence interval (CI) values for the WP as independent predictor of the composite endpoint are reported. The models for bivariate Cox regression analysis include alternatively age, estimated glomerular filtration rate (eGFR), N-terminal pro-B-type natriuretic peptide (NT-proBNP), left ventricular end-systolic diameter (LVESD), LV ejection fraction (LVEF), and late gadolinium enhancement (LGE)

$e G F R$ estimated glomerular filtration rate; $H R$ hazard ratio; $L G E$ late gadolinium enhancement; $L V E F$ left ventricular ejection fraction; LVESD left ventricular end-systolic diameter; NT-proBNP N-terminal pro-Btype natriuretic peptide
Funding Open access funding provided by Scuola Superiore Sant'Anna within the CRUI-CARE Agreement.

\section{Compliance with ethical standards}

Conflict of interest The authors declare that they have no conflict of interest to disclose.

Open Access This article is licensed under a Creative Commons Attribution 4.0 International License, which permits use, sharing, adaptation, distribution and reproduction in any medium or format, as long as you give appropriate credit to the original author(s) and the source, provide a link to the Creative Commons licence, and indicate if changes were made. The images or other third party material in this article are included in the article's Creative Commons licence, unless indicated otherwise in a credit line to the material. If material is not included in the article's Creative Commons licence and your intended use is not permitted by statutory regulation or exceeds the permitted use, you will need to obtain permission directly from the copyright holder. To view a copy of this licence, visit http://creativecommons.org/licenses/by/4.0/.

\section{References}

1. Westenberg JJ (2011) CMR for assessment of diastolic function. Curr Cardiovasc Imaging Rep 4(2):149-158

2. Rathi VK, Biederman RW (2009) Expanding role of cardiovascular magnetic resonance in left and right ventricular diastolic function. Heart Failure Clin 5(3):421-435

3. Barison A, Aimo A, Todiere G, Grigoratos C, Aquaro GD, Emdin M (2020) Cardiovascular magnetic resonance for the diagnosis and management of heart failure with preserved ejection fraction. Heart Failure Rev. https://doi.org/10.1007/s10741-020-09998-w

4. Sohal M, Duckett SG, Zhuang X, Shi W, Ginks M, Shetty A et al (2014) A prospective evaluation of cardiovascular magnetic resonance measures of dyssynchrony in the prediction of response to cardiac resynchronization therapy. J Cardiovasc Magn Reson Off J Soc Cardiovasc Magn Reson 16(1):58

5. Hor KN, Gottliebson WM, Carson C, Wash E, Cnota J, Fleck R et al (2010) Comparison of magnetic resonance feature tracking for strain calculation with harmonic phase imaging analysis. JACC Cardiovasc Imaging 3(2):144-151

6. Di Bella G, Minutoli F, Pingitore A, Zito C, Mazzeo A, Aquaro GD et al (2011) Endocardial and epicardial deformations in cardiac amyloidosis and hypertrophic cardiomyopathy. Circ J Off J Jpn Circ Soc 75(5):1200-1208 
7. Lima JA, Jeremy R, Guier W, Bouton S, Zerhouni EA, McVeigh E et al (1993) Accurate systolic wall thickening by nuclear magnetic resonance imaging with tissue tagging: correlation with sonomicrometers in normal and ischemic myocardium. J Am Coll Cardiol 21(7):1741-1751

8. Bilchick KC, Dimaano V, Wu KC, Helm RH, Weiss RG, Lima JA et al (2008) Cardiac magnetic resonance assessment of dyssynchrony and myocardial scar predicts function class improvement following cardiac resynchronization therapy. JACC Cardiovasc Imaging 1(5):561-568

9. Paelinck BP, de Roos A, Bax JJ, Bosmans JM, van Der Geest RJ, Dhondt D et al (2005) Feasibility of tissue magnetic resonance imaging: a pilot study in comparison with tissue Doppler imaging and invasive measurement. J Am Coll Cardiol 45(7):1109-1116

10. Petersen SE, Jung BA, Wiesmann F, Selvanayagam JB, Francis JM, Hennig J et al (2006) Myocardial tissue phase mapping with cine phase-contrast mr imaging: regional wall motion analysis in healthy volunteers. Radiology 238(3):816-826

11. Cao JJ, Ngai N, Duncanson L, Cheng J, Gliganic K, Chen Q (2018) A comparison of both DENSE and feature tracking techniques with tagging for the cardiovascular magnetic resonance assessment of myocardial strain. J Cardiovasc Magn Reson Off J Soc Cardiovasc Magn Reson 20(1):26

12. Osman NF, Sampath S, Atalar E, Prince JL (2001) Imaging longitudinal cardiac strain on short-axis images using strain-encoded MRI. Magn Reson Med 46(2):324-334

13. Soldo SJ, Norris SL, Gober JR, Haywood LJ, Colletti PM, Terk M (1994) MRI-derived ventricular volume curves for the assessment of left ventricular function. Magn Reson Imaging 12(5):711-717

14. McMurray JJ, Adamopoulos S, Anker SD, Auricchio A, Böhm M, Dickstein K et al (2012) ESC Guidelines for the diagnosis and treatment of acute and chronic heart failure 2012: the Task Force for the Diagnosis and Treatment of Acute and Chronic Heart Failure 2012 of the European Society of Cardiology. Developed in collaboration with the Heart Failure Association (HFA) of the ESC. Eur Heart J 33(14):1787-1847

15. Ponikowski P, Voors AA, Anker SD, Bueno H, Cleland JG, Coats $\mathrm{AJ}$ et al (2016) ESC Guidelines for the diagnosis and treatment of acute and chronic heart failure: the Task Force for the diagnosis and treatment of acute and chronic heart failure of the European Society of Cardiology (ESC). Developed with the special contribution of the Heart Failure Association (HFA) of the ESC. Eur J Heart Fail 18(8):891-975

16. Richardson P, McKenna W, Bristow M, Maisch B, Mautner B, O'Connell J et al (1996) Report of the 1995 World Health Organization/International Society and Federation of Cardiology Task Force on the Definition and Classification of cardiomyopathies. Circulation 93(5):841-842

17. Galeotti L, van Dam PM, Loring Z, Chan D, Strauss DG (2013) Evaluating strict and conventional left bundle branch block criteria using electrocardiographic simulations. Eur Eur Pacing arrhythm Cardiac Electrophysiol J Work Groups Cardiac Pacing Arrhythm Cardiac Cell Electrophysiol Eur Soc Cardiol 15(12):1816-1821

18. Lang RM, Badano LP, Mor-Avi V, Afilalo J, Armstrong A, Ernande L et al (2015) Recommendations for cardiac chamber quantification by echocardiography in adults: an update from the American Society of Echocardiography and the European Association of Cardiovascular Imaging. Eur Heart J Cardiovasc Imaging 16(3):233-270

19. Nagueh SF, Smiseth OA, Appleton CP, Byrd BF 3rd, Dokainish H, Edvardsen T et al (2016) Recommendations for the evaluation of left ventricular diastolic function by echocardiography: an update from the American Society of Echocardiography and the European Association of Cardiovascular Imaging. Eur Heart J Cardiovasc Imaging 17(12):1321-1360

20. Rudski LG, Lai WW, Afilalo J, Hua L, Handschumacher MD, Chandrasekaran K et al (2010) Guidelines for the echocardiographic assessment of the right heart in adults: a report from the American Society of Echocardiography endorsed by the European Association of Echocardiography, a registered branch of the European Society of Cardiology, and the Canadian Society of Echocardiography. J Am Soc Echocardiogr Off Publ Am Soc Echocardiogr 23(7):685-713

21. Lancellotti P, Tribouilloy C, Hagendorff A, Popescu BA, Edvardsen T, Pierard LA et al (2013) Recommendations for the echocardiographic assessment of native valvular regurgitation: an executive summary from the European Association of Cardiovascular Imaging. Eur Heart J Cardiovasc Imaging 14(7):611-644

22. Baumgartner H, Hung J, Bermejo J, Chambers JB, Evangelista A, Griffin BP et al (2009) Echocardiographic assessment of valve stenosis: EAE/ASE recommendations for clinical practice. J Am Soc Echocardiogr Off Publ Am Soc Echocardiogr 22(1):1-23

23. Emdin M, Passino C, Prontera C, Iervasi A, Ripoli A, Masini S et al (2004) Cardiac natriuretic hormones, neuro-hormones, thyroid hormones and cytokines in normal subjects and patients with heart failure. Clin Chem Lab Med 42(6):627-636

24. Passino C, Severino S, Poletti R, Piepoli MF, Mammini C, Clerico A et al (2006) Aerobic training decreases B-type natriuretic peptide expression and adrenergic activation in patients with heart failure. $\mathrm{J}$ Am Coll Cardiol 47(9):1835-1839

25. Lown B, Wolf M (1971) Approaches to sudden death from coronary heart disease. Circulation 44(1):130-142

26. Shephard DA (1976) The 1975 Declaration of Helsinki and consent. Can Med Assoc J 115(12):1191-1192

27. Nazarian S, Bluemke DA, Lardo AC, Zviman MM, Watkins SP, Dickfeld TL et al (2005) Magnetic resonance assessment of the substrate for inducible ventricular tachycardia in nonischemic cardiomyopathy. Circulation 112(18):2821-2825

28. Barison A, Aimo A, Ortalda A, Todiere G, Grigoratos C, Passino $\mathrm{C}$ et al (2018) Late gadolinium enhancement as a predictor of functional recovery, need for defibrillator implantation and prognosis in non-ischemic dilated cardiomyopathy. Int J Cardiol 250:195-200

29. Soriano CJ, Ridocci F, Estornell J, Jimenez J, Martinez V, De Velasco JA (2005) Noninvasive diagnosis of coronary artery disease in patients with heart failure and systolic dysfunction of uncertain etiology, using late gadolinium-enhanced cardiovascular magnetic resonance. J Am Coll Cardiol 45(5):743-748

30. Cerqueira MD, Weissman NJ, Dilsizian V, Jacobs AK, Kaul S, Laskey WK et al (2002) Standardized myocardial segmentation and nomenclature for tomographic imaging of the heart. A statement for healthcare professionals from the Cardiac Imaging Committee of the Council on Clinical Cardiology of the American Heart Association. Int J Cardiovasc Imaging 18(1):539-542

31. Hicks KA, Tcheng JE, Bozkurt B, Chaitman BR, Cutlip DE, Farb A et al (2015) 2014 ACC/AHA key data elements and definitions for cardiovascular endpoint events in clinical trials: a report of the American College of Cardiology/American Heart Association task force on clinical data standards (writing committee to develop cardiovascular endpoints data standardS). J Nucl Cardiol Off Publ Am Soc Nucl Cardiol 22(5):1041-1144

32. Vittinghoff E, McCulloch CE (2007) Relaxing the rule of ten events per variable in logistic and Cox regression. Am J Epidemiol 165(6):710-718

33. Thomas MC (2018) Perspective review: type 2 diabetes and readmission for heart failure. Clin Med Insights Cardiol 12:1179546818779588

34. Clark AL, Goode K, Cleland JG (2008) The prevalence and incidence of left bundle branch block in ambulant patients with chronic heart failure. Eur J Heart Fail 10(7):696-702

35. Bruch C, Stypmann J, Grude M, Gradaus R, Breithardt G, Wichter $\mathrm{T}$ (2006) Left bundle branch block in chronic heart failure-impact on diastolic function, filling pressures, and B-type natriuretic peptide levels. J Am Soc Echocardiogr Off Publ Am Soc Echocardiogr 19(1):95-101 
36. Ozdemir K, Altunkeser BB, Korkut B, Tokaç M, Gök H (2004) Effect of left bundle branch block on systolic and diastolic function of left ventricle in heart failure. Angiology 55(1):63-71

37. Francia P, Balla C, Paneni F, Volpe M (2007) Left bundle-branch block-pathophysiology, prognosis, and clinical management. Clin Cardiol 30(3):110-115

38. Pellicori P, Joseph AC, Zhang J, Lukaschuk E, Sherwi N, Bourantas CV et al (2015) The relationship of QRS morphology with cardiac structure and function in patients with heart failure. Clin Res Cardiol Off J German Cardiac Soc 104(11):935-945

39. Assomull RG, Shakespeare C, Kalra PR, Lloyd G, Gulati A, Strange $\mathrm{J}$ et al (2011) Role of cardiovascular magnetic resonance as a gatekeeper to invasive coronary angiography in patients presenting with heart failure of unknown etiology. Circulation 124(12):1351-1360

40. Gulati A, Jabbour A, Ismail TF, Guha K, Khwaja J, Raza S et al (2013) Association of fibrosis with mortality and sudden cardiac death in patients with nonischemic dilated cardiomyopathy. JAMA 309(9):896-908

41. Puntmann VO, Carr-White G, Jabbour A, Yu CY, Gebker R, Kelle S et al (2016) T1-mapping and outcome in nonischemic cardiomyopathy: all-cause mortality and heart failure. JACC Cardiovasc Imaging 9(1):40-50

42. Stankovic I, Prinz C, Ciarka A, Daraban AM, Kotrc M, Aarones M et al (2016) Relationship of visually assessed apical rocking and septal flash to response and long-term survival following cardiac resynchronization therapy (PREDICT-CRT). Eur Heart J Cardiovasc Imaging 17(3):262-269

Publisher's Note Springer Nature remains neutral with regard to jurisdictional claims in published maps and institutional affiliations. 